Proyecciones Journal of Mathematics

Vol. 36, No 3, pp. 511-527, September 2017.

Universidad Católica del Norte

Antofagasta - Chile

\title{
On fuzzy normed linear space valued statistically convergent sequences*
}

\author{
Paritosh Chandra Das \\ Rangia College, India \\ Received : January 2017. Accepted : April 2017
}

\begin{abstract}
In this article we define the notion of statistically convergent and statistically null sequences with the concept of fuzzy norm and discuss some of their properties such as completeness, monotone, solidness, symmetricity sequence algebra and convergence free.
\end{abstract}

Key Words : Fuzzy real number, statistical convergence, fuzzy normed linear space, monotone, solid space, symmetricity, sequence algebra and convergence free.

AMS Classification : 40A05, 40A35, 03E72.

${ }^{*}$ The work of the author is supported by University Grants Commission of India vide project No. F. 42-28/2013 (SR), dated 12 March, 2013, Rangia College, India 


\section{Introduction}

The concept of fuzzy set, a set whose boundary is not sharp or precise has been introduced by L. A. Zadeh in 1965. It is the origin of new theory of uncertainty, distinct from the notion of probability. After the introduction of fuzzy sets, the scope for studies in different branches of pure and applied mathematics increased widely. The notion of fuzzy sets has successfully been applied in studying sequence spaces with classical metric by Nanda [7], Savas [10], Tripathy and Baruah [15], Tripathy and Dutta [16] and many others. Using the concept of fuzzy metric, different authors namely Kelava and Seikkala [6], Syau [13] and many others have worked in different fields but, only a few works have been done on fuzzy norm by Das, Das and Choudhury [1], Esi [2], Felbin [4] and Tripathy and Nanda [18] and some others.

The notion of statistical convergence was introduced by Fast [3] and Schoenberg [11] independently. The potential of the introduced notion was realized in eighties by the workers on sequence spaces. Since than, a lot of work has been done on classical statistically convergent sequences. It is evidenced by the works of Fridy [5], Nuary and Savas [8], Šalát [9], Tripathy [14], Tripathy and Sen [17] and many others. There are many works done on statistically convergent sequences of fuzzy real numbers under classical metric, but we dont find work on statistically convergent sequences with the concept of fuzzy norm. A sizable work can be investigated in the direction of statistically convergent sequences with fuzzy norm.

\section{Definitions and preliminaries}

A fuzzy real number $X$ is a fuzzy set on $R$, i.e. a mapping $X: R \rightarrow I(=$ $[0,1])$ associating each real number $t$ with its grade of membership $X(t)$.

A fuzzy real number $X$ is called convex if $X(t) \geq X(s) \wedge X(r)=\min$ $(X(s), X(r))$, where $s<t<r$.

If there exists $t_{0} \in R$ such that $X\left(t_{0}\right)=1$, then the fuzzy real number $X$ is called normal.

A fuzzy real number $X$ is said to be upper-semi continuous if, for each 
$\varepsilon>0, X^{-1}([0, a+\varepsilon))$, for all $a \in I$ is open in the usual topology of $R$.

The set of all upper-semi continuous, normal, convex fuzzy real numbers is denoted by $R(I)$. Throughout the article, by a fuzzy real number we mean that the number belongs to $R(I)$.

The $\alpha$-level set $[X]^{\alpha}$ of the fuzzy real number $X$, for $0<\alpha \leq 1$, defined as $[X]^{\alpha}=\{t \in R: X(t) \geq \alpha\}$. If $\alpha=0$, then it is the closure of the strong 0 -cut. (The strong $\alpha$-cut of the fuzzy real number $X$, for $0 \leq \alpha \leq 1$ is the set $\{t \in R: X(t)>\alpha\})$.

For $X, Y \in R(I)$ consider a partial ordering $\leq$ as

$X \leq Y$ if and only if $a_{1}^{\alpha} \leq a_{2}^{\alpha}$ and $b_{1}^{\alpha} \leq b_{2}^{\alpha}$, for all $\alpha \in(0,1]$, where $[X]^{\alpha}=\left[a_{1}^{\alpha}, b_{1}^{\alpha}\right]$ and $[Y]^{\alpha}=\left[a_{2}^{\alpha}, b_{2}^{\alpha}\right]$.

Let $X, Y \in R(I)$ and $\alpha$-level sets be $[X]^{\alpha}=\left[a_{1}^{\alpha}, b_{1}^{\alpha}\right],[Y]^{\alpha}=\left[a_{2}^{\alpha}, b_{2}^{\alpha}\right]$, $\alpha \in[0,1]$. Then the arithmetic operations on $R(I)$ in terms of $\alpha$-level sets are defined as follows:

$$
\begin{aligned}
& {[X \oplus Y]^{\alpha}=\left[a_{1}^{\alpha}+a_{2}^{\alpha}, b_{1}^{\alpha}+b_{2}^{\alpha}\right],} \\
& {[X \ominus Y]^{\alpha}=\left[a_{1}^{\alpha}-b_{2}^{\alpha}, b_{1}^{\alpha}-a_{2}^{\alpha}\right],} \\
& {[X \otimes Y]^{\alpha}=\left[\min _{i, j \in\{1,2\}} a_{i}^{\alpha} b_{j}^{\alpha}, \max _{i, j \in\{1,2\}} a_{i}^{\alpha} b_{j}^{\alpha}\right]}
\end{aligned}
$$

and $\quad[\overline{1} \div Y]^{\alpha}=\left[\frac{1}{b_{2}^{\alpha}}, \frac{1}{a_{2}^{\alpha}}\right], 0 \notin Y$.

The absolute value, $|X|$ of $X \in R(I)$ is defined by (see for instance Kaleva and Seikkala [6])

$$
|X|(t)= \begin{cases}\max (X(t), X(-t)), & \text { for } t \geq 0 \\ 0, & \text { for } t<0 .\end{cases}
$$

A fuzzy real number $X$ is called non-negative if $X(t)=0$, for all $t<0$. The set of all non-negative fuzzy real numbers is denoted by $R^{*}(I)$.

\section{Statistically convergent sequence}

A subset $E$ of $N$ is said to have natural density $\delta(E)$ if 


$$
\delta(E)=\lim _{n \rightarrow \infty} \frac{1}{n} \sum_{k=1}^{\infty} \chi_{E}(k) \text { exists }
$$

where $\chi_{E}$ is the characteristic function of $E$. Clearly all finite subsets of $N$ have zero natural density and $\delta\left(E^{c}\right)=\delta(N-E)=1-\delta(E)$.

A given complex sequence $x=\left(x_{k}\right)$ is said to be statistically convergent to $L$ if for every $\varepsilon>0, \delta\left(\left\{k \in N:\left|x_{k}-L\right| \geq \varepsilon\right\}\right)=0$. We write $x_{k} \stackrel{\text { stat }}{\longrightarrow} L$ or stat-lim $x_{k}=L$.

Let $\left(x_{k}\right)$ and $\left(y_{k}\right)$ be two sequences, then we say that $x_{k}=y_{k}$ for almost all $k$ (in short a.a.k. ) if $\delta\left(k \in N: x_{k} \neq y_{k}\right)=0$.

\section{Fuzzy Normed Linear Space}

Let $X$ be a linear space over $R$ and the mapping $\|\cdot\|: X \rightarrow R^{*}(I)$ and the mappings $L, M:[0,1] \times[0,1] \rightarrow[0,1]$ be symmetric, non-decreasing in both arguments and satisfy $L(0,0)=0$ and $M(1,1)=1$. Write $[\|x\|]^{\alpha}=$ $\left[\left.\|\| x\left\|\left.\right|_{1} ^{\alpha},\right\|\|x\|\right|_{2} ^{\alpha},\right]$, for $x \in X, 0<\alpha \leq 1$ and suppose for all $x \in X, x \neq 0$, there exists $\alpha_{0} \in(0,1]$ independent of $x$ such that for all $\alpha \leq \alpha_{0}$,

(A) \|\|$x \|_{2}^{\alpha}<\infty$

(B) inf ${ }_{\alpha \in(0,1]}\left|\left\|x|\||_{1}^{\alpha}>0\right.\right.$.

The quadruple $(X,\|\cdot\|, L, M)$ is called a fuzzy normed linear space and $\|\cdot\|$ a fuzzy norm on the linear space $X$, if

i) $\|x\|=\overline{0}$ if and only if $x=0$,

ii) $\|r x\|=|r|\|x\|, x \in X, r \in R$,

iii) for all $x, y \in X$,

(a) $\|x+y\|(s+t) \geq L(\|x\|(s),\|y\|(t))$, whenever $s \leq\||x|\|_{1}^{1}, t \leq\|\| y \|_{1}^{1}$ and $s+t \leq\|\| x+y \|_{1}^{1}$,

(b) $\|x+y\|(s+t) \geq M(\|x\|(s),\|y\|(t))$, whenever $s \geq\|\mid\| x\left\|_{1}^{1}, t \geq\right\|\|y\|_{1}^{1}$ and $s+t \geq\|x+y\|_{1}^{1}$.

In the sequel we take $L(x, y)=\min (x, y)$ and $M(x, y)=\max (x, y)$ for $x, y \in[0,1]$ and we denote $(X,\|\cdot\|, L, M)$ by $(X,\|\cdot\|)$ or simply by $X$ in this case.

With these $L(x, y)=\min (x, y)$ and $M(x, y)=\max (x, y)$ for $x, y \in$ $[0,1]$, we have (refer to Felbin [4]) in a fuzzy normed linear space $(X,\|\cdot\|)$, 
the triangle inequality (iii) of the definition of fuzzy normed linear space is equivalent to

$$
\|x+y\| \leq\|x\| \oplus\|y\|
$$

The set $\omega(X)$ of all sequences in a vector space $X$ is a vector space with respect to pointwise addition and scalar multiplication. Any subspace $\lambda(X)$ of $\omega(X)$ is called vector valued sequence space. When $(X,\|\cdot\|)$ is a fuzzy normed linear space, then $\lambda(X)$ is called a fuzzy normed linear space-valued sequence space.

Throughout fnls denotes fuzzy normed linear space.

A fnls-valued sequence space $E^{F}(X)$ is said to be normal (or solid) if $\left(y_{k}\right) \in E^{F}(X)$, whenever $\left\|y_{k}\right\| \leq\left\|x_{k}\right\|$, for all $k \in N$ and $\left(x_{k}\right) \in E^{F}(X)$.

Let $K=\left\{k_{1}<k_{2}<k_{3} \ldots\right\} \subseteq N$ and $E^{F}(X)$ be a fnls-valued sequence space. A $K$-step space of $E^{F}(X)$ is a space of sequences $\lambda_{k}^{E^{F}}(X)=\left\{\left(x_{k_{n}}\right) \in\right.$ $\left.\omega^{F}(X):\left(x_{n}\right) \in E^{F}(X)\right\}$.

A canonical pre-image of a sequence $\left(x_{k_{n}}\right) \in \lambda_{k}^{E^{F}}(X)$ is a sequence $\left(y_{n}\right) \in \omega^{F}(X)$, defined as follows:

$$
y_{n}= \begin{cases}x_{n}, & \text { for } n \in K \\ \overline{0}, & \text { otherwise }\end{cases}
$$

A canonical pre-image of a step space $\lambda_{k}^{E^{F}}(X)$ is a space of canonical pre-images of all elements in $\lambda_{k}^{E^{F}}(X)$, i.e. $y$ is in canonical pre-image $\lambda_{k}^{E^{F}}(X)$ if and only if $y$ is canonical pre-image of some $x \in \lambda_{k}^{E^{F}}(X)$.

A fnls-valued sequence space $E^{F}(X)$ is said to be monotone if $E^{F}(X)$ contains the canonical pre-images of all its step spaces.

From the above definitions we have following remark.

Remark 2.1. A fnls-valued sequence space $E^{F}(X)$ is solid $\Rightarrow E^{F}(X)$ is monotone.

A fnls-valued sequence space $E^{F}(X)$ is said to be symmetric if $\left(x_{\pi(n)}\right) \in$ $E^{F}(X)$, whenever $\left(x_{k}\right) \in E^{F}(X)$, where $\pi$ is a permutation of $N$.

A fnls-valued sequence space $E^{F}(X)$ is said to be convergence free if $\left(y_{k}\right) \in E^{F}(X)$, whenever $\left(x_{k}\right) \in E^{F}(X)$ and $x_{k}=0$ implies $y_{k}=0$.

Let $(X,\|\cdot\|)$ be a fuzzy normed linear space. A sequence $\left(x_{n}\right) \in X$ is said to converge to $x \in X$, denoted by $\lim _{n \rightarrow \infty} x_{n}=x$, if and only if $\lim _{n \rightarrow \infty}\left\|x_{n}-x\right\|=\overline{0}$. 
i.e., $\lim _{n \rightarrow \infty}\left|\left\|x_{n}-x\left|\left\|_{1}^{\alpha}=\lim _{n \rightarrow \infty}\right\|\left\|x_{n}-x \mid\right\|_{2}^{\alpha}=0\right.\right.\right.$, for $\alpha \in(0,1]$.

Thus, $\lim _{n \rightarrow \infty}\left\|x_{n}-x\right\|=\overline{0}$ if and only if

$$
\lim _{n \rightarrow \infty}\|\| x_{n}-x \|_{2}^{\alpha}=0,
$$

for $0 \in(0,1]$.

A sequence $\left(x_{n}\right)$ in a fuzzy normed linear space $(X,\|\cdot\|)$ is called Cauchy if

$$
\lim _{\substack{n \rightarrow \infty \\ m \rightarrow \infty}}\left\|x_{m}-x_{n}\right\|=\overline{0}
$$

i.e., $\lim _{\substack{n \rightarrow \infty \\ m \rightarrow \infty}}\left|\left\|x_{n}-x \mid\right\|_{2}^{\alpha}=0\right.$, for $\alpha \in(0,1]$.

A fuzzy normed linear space $(X,\|\cdot\|)$ is called fuzzy complete if every Cauchy sequence in $X$ converges in $X$.

With the concept of fuzzy norm we define the following:

A sequence $\left(x_{k}\right)$ in a fuzzy norm linear space $(X,\|\cdot\|)$ is said to be statistically convergent to $x \in X$ if , for every $\bar{\varepsilon}>\overline{0}$,

$$
\delta\left(\left\{k \in N:\left\|x_{k}-x\right\| \geq \bar{\varepsilon}\right\}\right)=0
$$

and we write stat-lim $x_{k}=x$.

i.e., $\left(\delta\left(\left\{k \in N:\left\||| x_{k}-x\right\|_{1}^{\alpha} \geq \varepsilon\right\}\right)=0\right)=\left(\delta\left(\left\{k \in N:\left\|\mid x_{k}-x\right\|_{2}^{\alpha} \geq \varepsilon\right\}\right)=0\right)$, for $\alpha \in(0,1]$.

Thus, $\delta\left(\left\{k \in N:\left\|x_{k}-x\right\| \geq \varepsilon\right\}\right)=0$ if and only if $\delta\left(\left\{k \in N:\left\|\left|x_{k}-x\right|\right\|_{2}^{\alpha} \geq \varepsilon\right\}\right)=0$, for $\alpha \in(0,1]$.

A sequence $\left(x_{k}\right)$ in a fuzzy normed linear space $(X,\|\cdot\|)$ is said to be statistically null if $\left(x_{k}\right)$ statistically coverges to $0 \in X$.

Let $(X,\|\cdot\|)$ be a fuzzy normed linear space. Then for a sequence $x=\left(x_{k}\right) \in \bar{c}^{F}(X)$, the class of all statistically convergent sequences in $(X,\|\cdot\|)$ we define

$$
\|x\|=\sup _{k}\left\|x_{k}\right\|
$$

Clearly, $\|x\|$ is a norm. We also define the same norm for $\bar{c}_{0} F(X)$, the class of all statistically null sequences in $(X,\|\cdot\|)$ and $\ell_{\infty}^{F}(X)$, the class of all bounded sequences in $(X,\|\cdot\|)$. 
Throughout $\omega^{F}(X), \ell_{\infty}^{F}(X), c^{F}(X), c_{0}^{F}(X), \bar{c}^{F}(X), m^{F}(X), m_{0}^{F}(X)$

and $\bar{c}_{0} F(X)$ denote the class of all, bounded, convergent, null, statistically convergent, bounded statistically convergent, bounded statistically null and statistically null sequences in fuzzy normed linear space $X$ respectively.

\section{Main results}

Theorem 3.1. In a fuzzy normed linear space $(X,\|\cdot\|)$, the class of statistically convergent sequences, $\bar{c}^{F}(X)$ and the class of statistically null sequences, $\overline{c_{0}} F(X)$ are fuzzy normed linear space-valued sequence spaces.

Proof: We prove the result for the case of $\bar{c}^{F}(X)$. The other one can be established by similar technique.

Let $(X,\|\cdot\|)$ be a fnls and $x=\left(x_{k}\right), y=\left(y_{k}\right) \in \bar{c}^{F}(X)$.

Then for a given $\bar{\varepsilon}>\overline{0}$, there exists $l, m \in X$ such that

$$
\left\|x_{k}-l\right\|<\bar{\varepsilon}, \quad \text { for a.a.k. }
$$

and $\left\|y_{k}-m\right\|<\bar{\varepsilon}$ a.a.k.

We have $\left\|\left(x_{k}+y_{k}\right)-(l+m)\right\| \leq\left\|x_{k}-l\right\| \oplus\left\|y_{k}-m\right\|<\bar{\varepsilon}$, for a.a.k. Thus stat-lim $\left(x_{k}+y_{k}\right)=(l+m) \in X$ and hence $(x+y) \in \bar{c}^{F}(X)$. Again, for any $r \in R,\left\|r x_{k}-l\right\|=|r|\left\|x_{k}-l\right\|<\bar{\varepsilon}$, for a.a.k. Hence stat-lim $r x_{k}=r l$ and thus $r x \in \bar{c}^{F}(X)$. So, $\bar{c}^{F}(X)$ is a subspace of $\omega(X)$ and hence it is a fnls-valued sequence space.

Theorem 3.2. In a fuzzy normed linear space $(X,\|\cdot\|)$, the class of statistically bounded sequences, $m^{F}(X)=\bar{c}^{F}(X) \cap \ell_{\infty}^{F}(X)$ and $m_{0}^{F}(X)=$ ${\overline{c_{0}}}^{F}(X) \cap \ell_{\infty}^{F}(X)$ are closed subspaces of the complete fuzzy normed space $\ell_{\infty}^{F}(X)$ with the fuzzy norm

$$
\|x\|=\sup _{k}\left\|x_{k}\right\|
$$

where $x=\left(x_{k}\right)$ is in $m^{F}(X)$ or $m_{0}^{F}(X)$ and $X$ is complete. 
Proof: We prove the result for $m^{F}(X)$. The other one can be verified by similar technique. Since $X$ is complete. So it can be verified that $\ell_{\infty}^{F}(X)$ is complete. We show that $m^{F}(X)$ is complete with respect to given fuzzy norm. Let $\left(x^{(n)}\right)$ is a Cauchy sequence in $m^{F}$ and $x^{(n)} \rightarrow x$, as $n \rightarrow \infty$, say, in $\ell_{\infty}^{F}(X)$.

We show that $x \in m^{F}(X)$.

Since $x^{(n)}=\left(x_{k}^{(n)}\right)=\left(x_{1}^{(n)}, x_{2}^{(n)}, x_{3}^{(n)}, \ldots\right) \in m^{F}(X)$, so for each $n \in N$ there exists $a_{n} \in R$ such that

$$
\text { stat-lim } x_{k}^{(n)}=a_{n} .
$$

We prove the followings:

(i) $\lim _{n \rightarrow \infty} a_{n}=a$.

(ii) stat-lim $x_{k}=a$.

(i). Since $\left(x^{(n)}\right)$ is a convergent sequence, so for a given $\bar{\varepsilon}>\overline{0}$, there exists such a $n_{0} \in N$ that for each $m, n>n_{0}$ we have

$$
\begin{gathered}
\left\|x^{(m)}-x^{(n)}\right\|=\sup _{k}\left\|x_{k}^{(m)}-x_{k}^{(n)}\right\|<\frac{\bar{\varepsilon}}{3} . \\
\Rightarrow\left\|x_{k}^{(m)}-x_{k}^{(n)}\right\|<\frac{\bar{\varepsilon}}{3}, \quad \Rightarrow\|\| x_{k}^{(m)}-x_{k}^{(n)} \mid \|_{2}^{\alpha}<\frac{\varepsilon}{3},
\end{gathered}
$$

Again, since $x^{(n)}=\left(x_{k}^{(n)}\right) \in m^{F}(X)$, so for a given $\bar{\varepsilon}>\overline{0}$, we have

$$
\left\|x_{k}^{(m)}-a_{m}\right\|<\frac{\bar{\varepsilon}}{3}, \text { for a.a.k. } \Rightarrow\|\| x_{k}^{(m)}-a_{m} \|_{2}^{\alpha}<\frac{\varepsilon}{3}, \text { for a.a.k. }
$$

and

$$
\left\|x_{k}^{(n)}-a_{n}\right\|<\frac{\bar{\varepsilon}}{3}, \text { for a.a.k. } \Rightarrow\|\| x_{k}^{(n)}-a_{n} \|_{2}^{\alpha}<\frac{\varepsilon}{3}, \text { for a.a.k. }
$$

Now for each $m, n>n_{0} \in N$ and from the inequalities (3.1), (3.2) and (3.3), we get

$$
\left\|\left.\left|a_{m}-a_{n}\right|\right|_{2} ^{\alpha} \leq\right\||| a_{m}-x_{k}^{(m)}||_{2}^{\alpha}+\left.||\left|x_{k}^{(m)}-x_{k}^{(n)}\right|\right|_{2} ^{\alpha}+\left.||\left|x_{k}^{(n)}-a_{n}\right|\right|_{2} ^{\alpha} \text {, for a.a.k., }
$$
for all $\alpha \in(0,1]$.

$$
\Rightarrow\left\|\mid a_{m}-a_{n}\right\|_{2}^{\alpha}<\frac{\varepsilon}{3}+\frac{\varepsilon}{3}+\frac{\varepsilon}{3}=\varepsilon, \quad \Rightarrow\left\|a_{m}-a_{n}\right\|<\bar{\varepsilon}
$$


Thus $\left(a_{n}\right)$ is a Cauchy sequence in $X$. Since $X$ complete, so there exists such a number $a \in X$ such that

$$
\left\||| a_{m}-a_{n}||_{2}^{\alpha} \rightarrow 0, \quad \Rightarrow\right\| a_{n}-a \| \rightarrow \overline{0} \text { as } n \rightarrow \infty, \quad \Rightarrow \lim _{n \rightarrow \infty} a_{n}=a .
$$

(ii). We have $x^{(n)} \rightarrow x$. For a given $\bar{\xi}>\overline{0}$, there exists such a $q \in N$ that

$(3.4) \sup _{k}\left\|x_{k}^{(q)}-x_{k}\right\|<\frac{\bar{\xi}}{3}, \Rightarrow\left\|x_{k}^{(q)}-x_{k}\right\|<\frac{\bar{\xi}}{3}, \Rightarrow\|\| x_{k}^{(q)}-x_{k} \|_{2}^{\alpha}<\frac{\xi}{3}$.

The number $q$ can be chosen in such a way that together with (3.4), we get

$$
\left\|\left(a_{q}-a\right)\right\|<\frac{\bar{\xi}}{3} \Rightarrow\left\|\mid a_{q}-a\right\|_{2}^{\alpha}<\frac{\xi}{3 .}
$$

Since, stat-lim $x_{k}^{(q)}=a_{q}$.

For a given $\bar{\xi}>\overline{0},\left\|x_{k}^{(q)}-a_{q}\right\|<\frac{\bar{\xi}}{3}$, for a.a.k., for each fixed $q$.

$$
\Rightarrow\left\|\mid x_{k}^{(q)}-a_{q}\right\|_{2}^{\alpha}<\frac{\xi}{3}
$$

for a.a.k., for each fixed $q$

Now, $\left.||\left|x_{k}-a\right|\right|_{2} ^{\alpha} \leq\left|\left\|x_{k}-x_{k}^{(q)}||_{2}^{\alpha}+\left.||\left|x_{k}^{(q)}-a_{q}\right|\right|_{2} ^{\alpha}+\mid\right\| a_{q}-a \|_{2}^{\alpha}\right.$, for a.a.k.

$$
<\frac{\xi}{3}+\frac{\xi}{3}+\frac{\xi}{3}=\xi, \text { for a.a.k. } \Rightarrow\left\|x_{k}-a\right\|<\bar{\xi},
$$

for a.a.k.

Hence stat-lim $x_{k}=a$. This proves the result.

Theorem 3.3. (i) In a fuzzy normed linear space $(X,\|\cdot\|)$, the spaces $\bar{c}_{0}^{F}(X)$ and $m_{0}^{F}(X)$ are solid and as such are monotone.

(ii) In a fuzzy normed linear space $(X,\|\cdot\|)$, the spaces $\bar{c}^{F}(X)$ and $m^{F}(X)$ are neither monotone nor solid. 
Proof.(i) Consider two sequences $x=\left(x_{k}\right)$ and $y=\left(y_{k}\right)$ such that

$$
\left\|y_{k}\right\| \leq\left\|x_{k}\right\|
$$

for all $k \in N$ and let $\left(x_{k}\right) \in m_{0}^{F}(X) \subset \bar{c}_{0}^{F}(X)$.

Then for a given $\bar{\varepsilon}>\overline{0}$, we have

$$
\left\{k \in N:\left\|x_{k}\right\| \geq \bar{\varepsilon}\right\} \supseteq\left\{k \in N:\left\|y_{k}\right\| \geq \bar{\varepsilon}\right\} .
$$

Since $\left(x_{k}\right) \in m_{0}^{F}(X) \subset \bar{c}_{0}^{F}(X)$, so $\delta\left(\left\{k \in N:\left\|x_{k}\right\| \geq \bar{\varepsilon}\right\}\right)=0$

and hence $\delta\left(\left\{k \in N:\left\|y_{k}\right\| \geq \bar{\varepsilon}\right\}\right)=0$.

Thus $\left(y_{k}\right) \in m_{0}^{F}(X) \subset \bar{c}_{0}^{F}(X)$ and the class $\bar{c}_{0}^{F}(X)$ and $m_{0}^{F}(X)$ is solid.

The spaces ${\overline{c_{0}}}^{F}(X)$ and $m_{0}^{F}(X)$ are monotone follows from Remark 2.1.

Proof.(ii) The result follows from the following example.

Example 3.1. Consider the sequence $x=\left(x_{k}\right)$ defined as follows.

$$
x_{k}= \begin{cases}1, & \text { for } \quad k=n^{2}, n \in N \\ 2-\frac{1}{k}, & \text { for } \quad k \neq n^{2}, n \in N .\end{cases}
$$

and $\|x\|=\sup _{k}\left\|x_{k}\right\|$.

We have $X$ is a fuzzy normed linear space. For any sequence $z=\left(z_{k}\right) \in$ $X$, let us consider $\left\|z_{k}\right\|$, defined as follows:

(A) For $\mathrm{k} \in N, z_{k} \neq 0,\left\|z_{k}\right\|(t)= \begin{cases}\frac{2 t}{\left|z_{k}\right|}-1, & \text { for } \frac{\left|z_{k}\right|}{2} \leq t \leq\left|z_{k}\right| \\ 0, & \text { otherwise }\end{cases}$ and for $z_{k}=0,\left\|z_{k}\right\|(t)= \begin{cases}1, & \text { for } t=0 \\ 0, & \text { otherwise. }\end{cases}$

Using (A), we have for $k=n^{2}, n \in N$,

$$
\left\|x_{k}\right\|(t)= \begin{cases}\frac{2 t}{\left|x_{k}\right|}-1, & \text { for } \frac{\left|x_{k}\right|}{2} \leq t \leq\left|x_{k}\right|=1, \\ 0, & \text { otherwise. }\end{cases}
$$


and for $k \neq n^{2}, n \in N$,

$$
\left\|x_{k}\right\|(t)= \begin{cases}\frac{2 t}{\left|x_{k}\right|}-1, & \text { for } \frac{\left|x_{k}\right|}{2} \leq t \leq\left|x_{k}\right|=2-\frac{1}{k} \\ 0, & \text { otherwise. }\end{cases}
$$

Therefore, $\left\|x_{k}\right\|(t)=\sup _{k}\left\|x_{k}\right\|(t)= \begin{cases}t-1, & \text { for } \frac{\left|x_{k}\right|}{2} \leq t \leq\left|x_{k}\right|=2, \\ 0, & \text { otherwise. }\end{cases}$

Thus $\left\|x_{k}\right\|$ is bounded. $(A))$,

Again, we have for a given $\bar{\varepsilon}>\overline{0}\left(\bar{\varepsilon}\right.$ is a fuzzy no. as defined $\left\|z_{k}\right\|$ in

$\left\|x_{k}-2\right\|<\bar{\varepsilon}$, for a.a.k. (i.e., $\left.k \neq n^{2}\right)$.

$\Rightarrow$ stat-lim $\left\|x_{k}-2\right\|=\overline{0}$. Thus stat-lim $x_{k}=2$ w.r.t. the fuzzy norm. Hence $\left(x_{k}\right) \in m^{F}(X) \subset \bar{c}^{F}(X)$.

Let $J=\{k \in N: k=2 i, i \in N\}$ be a subset of $N$ and let $\overline{\overline{\left(m^{F}(X)\right)}}$ be the canonical pre-image of the $J$-step space $\left(m^{F}(X)\right)_{J}$ of $m^{F}(X)$, defined as follows:

$\left(y_{k}\right) \in{\overline{\overline{\left(m^{F}(X)\right)}}}_{J}$ is the canonical pre-image of $\left(x_{k}\right) \in m^{F}(X)$ implies

$$
y_{k}= \begin{cases}x_{k}, & \text { for } k \in J \\ \overline{0} & \text { for } k \notin J\end{cases}
$$

Now, for $k \in J$ and $k=n^{2}, n \in N$ and using (3.5) we have

$$
\left\|y_{k}\right\|(t)= \begin{cases}\frac{2 t}{\left|x_{k}\right|}-1, & \text { for } \frac{\left|x_{k}\right|}{2} \leq t \leq\left|x_{k}\right|=1 \\ 0, & \text { otherwise. }\end{cases}
$$

i.e., We have $\left\|y_{k}-1\right\|=\overline{0}$, for infinitely many $k$.

Again, for $k \in J$ and $k \neq n^{2}, n \in N$ and using (3.6) we have

$$
\left\|y_{k}\right\|(t)= \begin{cases}\frac{2 t}{\left|x_{k}\right|}-1, & \text { for } \frac{\left|x_{k}\right|}{2} \leq t \leq\left|x_{k}\right|=2-\frac{1}{k} \\ 0, & \text { otherwise. }\end{cases}
$$

i.e., For a given $\bar{\varepsilon}>\overline{0}$, we have $\left\|y_{k}-2\right\|<\bar{\varepsilon}$, for infinitely many $k(\bar{\varepsilon}$ is a fuzzy no. as defined $\left\|z_{k}\right\|$ in $\left.(A)\right)$. 
For $k \notin J,\left\|y_{k}\right\|(t)= \begin{cases}1, & \text { for } t=0, \\ 0, & \text { otherwise. }\end{cases}$

i.e., We have $\left\|y_{k}-0\right\|=\overline{0}$, for infinitely many $k$.

Therefore, for a given $\varepsilon>0$ and $\alpha \in(0,1]$, there is no definite point, say L such that

$$
\left\|\mid y_{k}-L\right\|_{2}^{\alpha}<\varepsilon
$$

for a.a.k.

i.e., $\left\|y_{k}-L\right\|<\bar{\varepsilon}$, for given $\bar{\varepsilon}>\overline{0}$ and for a.a.k.

Thus $\left(y_{k}\right) \notin \bar{c}^{F}(X)\left(\supset m^{F}(X)\right)$. Hence $\bar{c}^{F}(X)$ and $m^{F}(X)$ are not monotone.

The spaces $\bar{c}^{F}(X)$ and $m^{F}(X)$ are not solid follows from the Remark 2.1.

Theorem 3.4. The spaces $\bar{c}^{F}(X), \bar{c}_{0}^{F}(X), m^{F}(X)$ and $m_{0}{ }^{F}(X)$ are not symmetric.

Proof. The result follows from the following example.

Example 3.2. Consider the sequence $x=\left(x_{k}\right)$ defined as follows.

$$
x_{k}= \begin{cases}1, & \text { for } k=n^{2}, n \in N \\ \frac{1}{k}, & \text { for } k \neq n^{2}, n \in N\end{cases}
$$

and $\|x\|=\sup _{k}\left\|x_{k}\right\|$.

Using (A) of Example 3.1, we have for $k=n^{2}, n \in N$,

$$
\left\|x_{k}\right\|(t)= \begin{cases}\frac{2 t}{\left|x_{k}\right|}-1, & \text { for } \frac{\left|x_{k}\right|}{2} \leq t \leq\left|x_{k}\right|=1, \\ 0, & \text { otherwise. }\end{cases}
$$

and for $k \neq n^{2}, n \in N, \quad\left\|x_{k}\right\|(t)= \begin{cases}\frac{2 t}{\left|x_{k}\right|}-1, & \text { for } \frac{\left|x_{k}\right|}{2} \leq t \leq\left|x_{k}\right|=\frac{1}{k}, \\ 0, & \text { otherwise. }\end{cases}$

Therefore, $\left\|x_{k}\right\|(t)=\sup _{k}\left\|x_{k}\right\|(t)= \begin{cases}2 t-1, & \text { for } \frac{\left|x_{k}\right|}{2} \leq t \leq\left|x_{k}\right|=1, \\ 0, & \text { otherwise. }\end{cases}$ 
Thus $\left\|x_{k}\right\|$ is bounded.

Again, we have for a given $\bar{\varepsilon}>\overline{0}\left(\bar{\varepsilon}\right.$ is a fuzzy no. as defined $\left\|z_{k}\right\|$ in $\left.(A)\right)$, $\left\|x_{k}-0\right\|<\bar{\varepsilon}$, for a.a.k. (i.e., $\left.k \neq n^{2}\right)$.

$\Rightarrow$ stat-lim $\left\|x_{k}-0\right\|=\overline{0}$.

Thus stat-lim $x_{k}=0$ w.r.t. the fuzzy norm. Hence $\left(x_{k}\right) \in H$, for $H=$ $\bar{c}^{F}(X), \bar{c}_{0} F(X), m^{F}(X)$ and $m_{0} F(X)$.

Let $\left(y_{k}\right)$ be a rearrangement of the sequence $\left(x_{k}\right)$, defined as follows.

$\left(y_{k}\right)=\left(x_{1}, x_{2}, x_{4}, x_{3}, x_{9}, x_{5}, x_{16}, x_{6}, x_{25}, x_{7}, \ldots\right)$.

i.e.,

$\left(y_{k}\right)= \begin{cases}x_{\left(\frac{k+1}{2}\right)^{2},}, & \text { for } k \text { odd, } \\ x_{\left(n+\frac{k}{2}\right)}, & \text { for } k \text { even and } n \in N, \text { satisfies } n(n-1)<\frac{k}{2} \leq n(n+1) .\end{cases}$

Thus $\left(y_{k}\right)=\left(1, \frac{1}{2}, 1, \frac{1}{3}, 1, \frac{1}{5}, 1, \frac{1}{6}, 1, \frac{1}{7}, 1, \frac{1}{8}, 1, \frac{1}{10}, 1, \frac{1}{11}, \ldots\right)$.

Using $(A)$ of Example 3.1, we have for $k$ odd,

$\left\|y_{k}\right\|(t)=\left\|x_{\left(\frac{k+1}{2}\right)^{2}}\right\|(t)= \begin{cases}\frac{2 t}{\mid x_{\left(\frac{k+1}{2}\right)^{2}}}-1, & \text { for } \frac{\mid x_{\left(\frac{k+1}{2}\right)^{2} \mid}^{2}}{2} \leq t \leq\left|x_{\left(\frac{k+1}{2}\right)^{2}}\right|=1, \\ 0, & \text { otherwise. }\end{cases}$

and for $k$ even ; $n \in N$, satisfying $n(n-1)<\frac{k}{2} \leq n(n+1)$,

$$
\left\|y_{k}\right\|(t)=\left\|x_{\left(n+\frac{k}{2}\right)}\right\|(t)= \begin{cases}\frac{2 t}{\mid x_{\left(n+\frac{k}{2}\right)}}-1, & \text { for } \frac{\left|x_{\left(n+\frac{k}{2}\right)}\right|}{2} \leq t \leq\left|x_{\left(n+\frac{k}{2}\right)}\right|=\frac{1}{\left(n+\frac{k}{2}\right)}, \\ 0, & \text { otherwise. }\end{cases}
$$

Now, for a given $\varepsilon>0$ and $\alpha \in(0,1]$, there is no definite point, say $L$ such that

$$
\left\|y_{k}-L\right\|_{2}^{\alpha}<\varepsilon
$$

for a.a.k.

i.e., There is no definite $L$ such that $\left\|y_{k}-L\right\|<\bar{\varepsilon}$, for given $\bar{\varepsilon}>\overline{0}$ and for a.a.k.

Thus $\left(y_{k}\right) \notin H$, for $H=\bar{c}^{F}(X),{\overline{c_{0}}}^{F}(X), m^{F}(X)$ and $m_{0} F(X)$. Hence $\bar{c}^{F}(X),{\overline{c_{0}}}^{F}(X), m^{F}(X)$ and $m_{0} F(X)$ are not symmetric.

Theorem 3.5. The spaces $\bar{c}^{F}(X), \bar{c}_{0} F(X), m^{F}(X)$ and $m_{0}{ }^{F}(X)$ are not convergence free.

Proof: The result follows from the following example. 
Example 3.3. Consider the sequence $x=\left(x_{k}\right)$ defined as follows.

$$
x_{k}= \begin{cases}0, & \text { for } k=n^{2}, n \in N \\ k^{-1}, & \text { for } k \neq n^{2}, n \in N .\end{cases}
$$

Consider, $\|x\|=\sup _{k}\left\|x_{k}\right\|$. Using (A) of Example 3.1, we have for $k=$ $n^{2}, n \in N$,

$$
\left\|x_{k}\right\|(t)= \begin{cases}1, & \text { for } t=0 \\ 0, & \text { otherwise. }\end{cases}
$$

and for $k \neq n^{2}, n \in N, \quad\left\|x_{k}\right\|(t)= \begin{cases}\frac{2 t}{\left|x_{k}\right|}-1, & \text { for } \frac{\left|x_{k}\right|}{2} \leq t \leq\left|x_{k}\right|=k^{-1}, \\ 0, & \text { otherwise. }\end{cases}$

Therefore, $\left\|x_{k}\right\|(t)=\sup _{k}\left\|x_{k}\right\|(t)= \begin{cases}4 t-1, & \text { for } \frac{\left|x_{k}\right|}{2} \leq t \leq\left|x_{k}\right|=\frac{1}{2}, \\ 0, & \text { otherwise. }\end{cases}$ Thus $\left\|x_{k}\right\|$ is bounded.

Again, we have for a given $\bar{\varepsilon}>\overline{0}\left(\bar{\varepsilon}\right.$ is a fuzzy no. as defined $\left\|z_{k}\right\|$ in $(A))$

$\left\|x_{k}-0\right\|<\bar{\varepsilon}$, for a.a.k. (i.e., $k \neq n^{2}$ ).

$\Rightarrow$ stat-lim $\left\|x_{k}-0\right\|=\overline{0}$.

Thus stat-lim $x_{k}=0$ w.r.t. the fuzzy norm. Hence $\left(x_{k}\right) \in H$, for $H=\bar{c}^{F}(X),{\overline{c_{0}}}^{F}(X), m^{F}(X)$ and $m_{0} F(X)$.

Let the sequence $\left(y_{k}\right)$ be defined as follows:

$$
y_{k}= \begin{cases}0, & \text { for } k=n^{2}, n \in N, \\ 3, & \text { for } k \neq n^{2}, n \in N \text { and } k \text { odd } \\ 2, & \text { for } k \neq n^{2}, n \in N \text { and } k \text { even }\end{cases}
$$

Using (A) of Example 3.1, we have for $k=n^{2}, n \in N$,

$$
\left\|y_{k}\right\|(t)= \begin{cases}1, & \text { for } t=0 \\ 0, & \text { otherwise }\end{cases}
$$

and for $k \neq n^{2}, n \in N$ and $k$ odd,

$$
\left\|y_{k}\right\|(t)= \begin{cases}\frac{2 t}{\left|y_{k}\right|}-1, & \text { for } \frac{\left|y_{k}\right|}{2} \leq t \leq\left|y_{k}\right|=3 \\ 0, & \text { otherwise. }\end{cases}
$$


i.e., We have $\left\|y_{k}-3\right\|=\overline{0}$, for infinitely many $k$ and for $k \neq n^{2}, n \in N$ and $k$ even,

$$
\left\|y_{k}\right\|(t)= \begin{cases}\frac{2 t}{\left|y_{k}\right|}-1, & \text { for } \frac{\left|y_{k}\right|}{2} \leq t \leq\left|y_{k}\right|=2, \\ 0, & \text { otherwise. }\end{cases}
$$

i.e., We have $\left\|y_{k}-2\right\|=\overline{0}$, for infinitely many $k$.

Now, for a given $\varepsilon>0$ and $\alpha \in(0,1]$, there is no definite point, say L such that

$$
\left\|\mid y_{k}-L\right\|_{2}^{\alpha}<\varepsilon
$$

for a.a.k.

i.e., There is no definite $L$ such that $\left\|y_{k}-L\right\|<\bar{\varepsilon}$, for given $\bar{\varepsilon}>\overline{0}$ and for a.a.k.

Thus $\left(y_{k}\right) \notin H$, for $H=\bar{c}^{F}(X), \bar{c}_{0}^{F}(X), m^{F}(X)$ and $m_{0} F(X)$. Hence $\bar{c}^{F}(X),{\overline{c_{0}}}^{F}(X), m^{F}(X)$ and $m_{0} F(X)$ are not convergence free.

Theorem 3.6. The spaces $\bar{c}^{F}(X), \bar{c}_{0}^{F}(X), m^{F}(X)$ and $m_{0} F(X)$ are sequence algebra.

Proof. We prove this for $m_{0} F(X)$ and the other spaces can be proved by similar method.

Let $\overline{0}<\bar{\varepsilon}<\overline{1}$ be given. Suppose $x=\left(x_{k}\right), y=\left(y_{k}\right) \in m_{0}{ }^{F}(X)$. Consider $\|x\|=\sup _{k}\left\|x_{k}\right\|$ and $\|x \times y\|=\sup _{k}\left\|x_{k} \times y_{k}\right\|$.

Since $\left(x_{k}\right),\left(y_{k}\right) \in m_{0} F(X)$. Then we have,

$$
\begin{aligned}
& \left\{k \in N:\left\|x_{k} \times y_{k}\right\|<\bar{\varepsilon}\right\} \supseteq\left\{k \in N:\left\|x_{k}\right\|<\sqrt{\bar{\varepsilon}}\right\} \cap\left\{k \in N:\left\|y_{k}\right\|<\sqrt{\bar{\varepsilon}}\right\} \\
& \text { Since } \delta\left(\left\{k \in N:\left\|x_{k}\right\|<\sqrt{\bar{\varepsilon}}\right\}\right)=1, \quad \delta\left(\left\{k \in N:\left\|y_{k}\right\|<\sqrt{\bar{\varepsilon}}\right\}\right)=1 \\
& \text { So, } \delta\left(\left\{k \in N:\left\|x_{k} \times y_{k}\right\|<\bar{\varepsilon}\right\}\right)=1
\end{aligned}
$$

Thus $\left(x_{k} \times y_{k}\right) \in m_{0} F(X)$. Hence the space $m_{0} F(X)$ is sequence algebra. 


\section{References}

[1] N. R. Das, P. Das and A. Choudhury: Absolute value like fuzzy real number and fuzzy real-valued sequence spaces, Jour. Fuzzy Math., 4(2), pp. 421-433, (1996).

[2] A. Esi, : On some new paranormed sequence spaces of fuzzy numbers defined by Orlicz functions and statistical convergence, Math. Modell. Anal., 11(4), pp. 379-388, (2006).

[3] H. Fast: Sur la convergence statistique, Colloq. Math., 2, pp. 241-244, (1951).

[4] C. Felbin: Finit dimensional fuzzy normed linear space, Fuzzy Sets Syst., 48, pp. 239-248, (1992).

[5] J. A. Fridy: On statistical convergence, Analysis, 5, pp. 301-313, (1985).

[6] O. Kelava and S. Seikkala: On fuzzy metric spaces, Fuzzy Sets Syst., 12, pp. 215-229, (1984).

[7] S. Nanda: On sequences of fuzzy numbers, Fuzzy Set Syst., 33, pp. 123-126, (1989).

[8] F. Nuray and E. Savas: Statistical convergence of sequences of fuzzy real numbers, Math. Slovaca, 45 (3), pp. 269-273, (1995).

[9] T. Šalát: On Statistically convergent sequences of real numbers, Math. Slovaca, 30 (2), pp. 139-150, (1980).

[10] E. Savas: A note on sequence of fuzzy numbers, Inf. Sci., 124, pp. 297-300, (2000).

[11] I. J. Schoenberg: The integrability of certain functions and related summability methods, Amer. Math. Monthly, 66, pp. 361-375, (1959).

[12] P. V. Subrahmanyam: Cesàro Summability for fuzzy real numbers;, J. Analysis, 7, pp. 159-168, (1999).

[13] Yu-R. Syau: Sequences in fuzzy metric space, Computers Math. Appl., 33(6), pp. 73-76, (1997). 
[14] B. C. Tripathy: On generalized difference paranormed statistically convergent sequences, Indian J. Pure Appl. Math., 35(5), pp. 655-663, (2004).

[15] B. C. Tripathy and A. Baruah: Lacunary statistically convergent and lacunary strongly convergent generalized difference sequences of fuzzy real numbers, Kyungpook Math. J., 50, pp. 565-574, (2010).

[16] B. C. Tripathy and A.J. Dutta: Bounded variation double sequence space of fuzzy real numbers, Comput. Math. Appl., 59 (2), pp. 10311037, (2010).

[17] B. C. Tripathy and M. Sen: On generalized statistically convergent sequences, Indian J. Pure Appl. Math., 32(11), pp. 1689-1694, (2001).

[18] B. K. Tripathy and S. Nanda: Absolute value of fuzzy real numbers and fuzzy sequence spaces, Jour. Fuzzy Math., 8(4), pp. 883-892, (2000).

\section{Paritosh Chandra Das}

Department of Mathematics

Rangia College,

Rangia-781354; Assam, INDIA

e-mail : daspc_rangia@yahoo.com; pcdasrc2011@gmail.com 